\title{
Hallucinations and Covid-19: Increased Occurrence of Hallucinations in Patients with Alzheimer's Disease During Lockdown
}

\author{
Mohamad El Haj ${ }^{1,2,3}$ (D) Frank Larøi ${ }^{4,5,6} \cdot$ Karim Gallouj $^{2}$
}

Accepted: 30 May 2021 / Published online: 5 June 2021

(C) The Author(s), under exclusive licence to Springer Science+Business Media, LLC, part of Springer Nature 2021

\begin{abstract}
We investigated the effects of lockdown, as implemented by retirement homes to cope with the spread of Covid-19, on hallucinatory experiences in patients with Alzheimer's disease (AD). The study included 47 patients with AD living in retirement homes and who were already experiencing hallucinations prior to the lockdown. We invited caregivers to rate hallucinatory experiences in these patients during the lockdown, and compared this rating with that provided by the same caregivers prior to the lockdown. Results demonstrated increased hallucinatory experiences in patients with AD during the lockdown, compared with before the lockdown. The decrease in social and physical activities during the lockdown, and especially, the physical separation of residents from family members, might have led to decreased sensory stimulation and increased loneliness, and consequently, to the hallucinatory experiences in patients with $\mathrm{AD}$ living in retirement homes during the lockdown. While the restrictive measures were necessary to cope with the spread of Covid19 , these measures have increased hallucinations in patients with AD living in retirement homes, at least in those who were already experiencing hallucinations prior to the lockdown.
\end{abstract}

Keywords Alzheimer's disease $\cdot$ Covid-19 $\cdot$ Hallucinations $\cdot$ Lockdown

Mohamad El Haj

mohamad.elhaj@univ-nantes.fr

1 Nantes Université, Univ Angers, Laboratoire de Psychologie Des Pays de La Loire (LPPL - EA 4638), 44000 Nantes, France

2 Unité de Gériatrie, Centre Hospitalier de Tourcoing, Tourcoing, France

3 Institut Universitaire de France, Paris, France

4 Department of Biological and Medical Psychology, University of Bergen, Bergen, Norway

5 Norwegian Center of Excellence for Mental Disorders Research, University of Oslo, Oslo, Norway

6 Psychology and Neuroscience of Cognition Research Unit, University of Liège, Liège, Belgium 
The Covid-19 crisis has radically changed our social life and entirely altered how retirement homes provide care to residents, especially to those with Alzheimer's disease (AD) [1]. Because patients with AD have a high risk of Covid-19 infection, retirement homes were forced to implement strict restrictive measures. While these measures have served to limit the spread of Covid-19, these measures involved reduced physical contact between residents and their families. These restrictive measures also involved the cancelation of several internal and external activities, not only those considered as nonessential such as leisure activities (e.g., hairdressing), but also those involving access to health services resources (e.g., access to external memory clinics, diurnal centers, physiotherapy centers). While these measures were intended to protect the residents against Covid-19, these measures have increased isolation and loneliness in the residents and, probably, have decreased their overall wellbeing and mental health. Critically, as will be discussed in the next paragraphs, these restrictive measures might have increased the occurrence of hallucinations in patients with $\mathrm{AD}$, especially in those already suffering from hallucinations prior to the lockdown.

Before discussing how the lockdown might have increased hallucinations in patients with $\mathrm{AD}$, it would be of interest to provide an introduction on the literature on hallucinations in $\mathrm{AD}$. Although $\mathrm{AD}$ is mainly characterized by cognitive decline [2], the disease is also characterized by psychiatric symptoms such as hallucinations $[3,4]$. The prevalence of hallucinations in $\mathrm{AD}$ can range from 4 to $76 \%$ (median 23\%) [5] with increased occurrence in patients with moderate or advanced AD [6]. Although hallucinations in AD mainly contain visual and auditory elements (e.g., faces or voices), other sensory (e.g., olfactory, somatic, or tactile) elements can sometimes be evoked by patients during hallucinations [3, 4, 7]. At the cognitive level, hallucinations in AD can be associated with declines in source monitoring, i.e., the ability of patients to determine whether an experience originated within the self or came from an external source [8]. Hallucinations in AD can also be attributed to inhibitory dysfunction which leads to the emergence of redundant or irrelevant information into awareness, leading to hallucinations $[9,10]$. Besides cognitive factors, hallucinations in AD can also be associated with neurological factors such as reduced thickness in the lateral parietal cortex [11], an increased occipital periventricular hyperintensities [12], as well as with low regional perfusion of the right and left dorsolateral frontal, left ventral striatal, left anterior cingulate, left pulvinar and dorsolateral parietal cortex regions [13]. Regardless of their cognitive or neurological underlying processes, hallucinations in $\mathrm{AD}$ can lead to aggressive behavior [14], verbal outbursts [15], and functional decline [16-18]. In turn, aggressive behavior and functional decline, due to hallucinations, often lead to stress, depression, and a high level of burden of care in caregivers [19, 20].

Hallucinations in patients with $\mathrm{AD}$, especially in those living in retirement homes, might have increased during the lockdown. This assumption can be supported be research demonstrating how the lockdown has resulted in decreased mental health of patients with AD. For instance, research has demonstrated increased neuropsychiatric symptomatology in patients with AD living in their own homes during the lockdown [21]. Regarding patients with $\mathrm{AD}$ who live in retirement homes, research has demonstrated increased loneliness [22-24] and depression in these patients during the lockdown [25]. Research has also reported case studies involving hallucinations in three patients with AD who live in retirement homes during the Covid-19 crisis [26, 27]. These case studies have included patients who presented hallucinations prior to the lockdown and compared the patients' hallucination scores before and during the lockdown. These case studies have reported increased hallucinations during the lockdown and these increased hallucinations were 
attributed to the social distancing, lack of physical contact with family members and loneliness of patients during the lockdown.

While the above-mentioned case studies have reported how the lockdown has increased hallucinations in patients with AD living in retirement homes, these reports are preliminary and should be tested in a more representative sample. We thus investigated hallucinations in a large sample of patients with $\mathrm{AD}$ living in retirement homes during the lockdown. We were also inspired by a study by Cagnin et al. [28] who assessed hallucinations in a large sample of patients with $\mathrm{AD}$ who were living in their own homes during the lockdown. In their study, Cagnin et al. [28] invited family members of patients with AD to describe changes in neuropsychiatric symptoms of the patients during the lockdown. Results demonstrated increased hallucinations in some patients with AD during the lockdown. While the study by Cagnin et al. [28] has the merit to assess hallucinations in a very large sample of patients with $\mathrm{AD}(n=3372)$, this study lacked a baseline, i.e., an assessment of hallucinations in patients prior to lockdown. Critically, this study was also concerned with patients living in their own homes and not those living in retirement homes. Targeting patients living in retirement homes is important because, unlike patients living in their own homes with their family members, patients living in retirement homes have suffered limited physical contact with their family members during the lockdown. Also, compared with patients living in their own homes, those living in retirement homes are typically in the advanced stages of the disease and suffer more cognitive and functional decline, thus exacerbating their neuropsychiatric symptomatology. We therefore assessed hallucinations in patients with $\mathrm{AD}$ living in retirement homes during the lockdown.

To summarize, the restrictive measures during the lockdown have decreased wellbeing and mental health in patients with AD living in their own homes [21, 28] as well as in patients living in retirement homes [22-25]. These restrictive measures have also increased hallucinations as suggested by research reporting how the lockdown has increased hallucinations in patients with AD living in retirement homes [26, 27], however, these findings were based on case studies and should be tested in a more representative sample. We thus investigated hallucinations in a large sample of patients with AD living in retirement homes during the lockdown. We used a follow-up design in which we compared hallucinations scores in patients with $\mathrm{AD}$ living in retirement homes and presenting hallucinations prior to the lockdown. More specifically, we compared hallucination scores of these patients during the lockdown and before the lockdown. We expected a higher occurrence of hallucination during the lockdown that before the lockdown in these patients.

\section{Method}

\section{Participants}

The study included forty-seven participants with a clinical diagnosis of probable AD as defined by the National Institute on Aging and the Alzheimer's Association criteria for probable Alzheimer's disease [2] (27 women and 20 men; $M$ age $=71.91$ years, $S D=5.33$; $M$ years of formal education $=7.81, S D=2.28$ ). We recruited participants from a pool of patients who already participated in our previous studies on hallucinations before the lockdown [4, 7-9]. Participants were living in different retirement homes in France and their mean score on the Mini Mental Stat Examination [29] was 14.30 ( $S D=2.06)$. We determined the sample size with $G^{*}$ Power [30], we calculated sample size for Wilcoxin 
tests (see the results section where we explain why we used Wilcoxin tests) based on $95 \%$ power, an estimated probability of making Type I error of 0.05 , and a medium effect size of 0.50 [31]. Analysis suggested that 47 participants would be necessary to obtain sufficient statistical power. This study was designed and conducted in accordance with the Declaration of Helsinki and participants provided their consent to participate and were able to withdraw whenever they wished.

\section{Procedures}

Generally speaking, we invited caregivers to rate hallucinations of patients during the confinement, and we compared this rating with that already provided by the same caregivers prior to the lockdown. The caregivers were either psychologists or nurses who provided care for the patients both before and during the lockdown. During the lockdown, we invited the caregivers to rate hallucinations of patients using the three items, the same as those used by the caregivers to rate hallucinations before the lockdown in our studies [4, 7-9]. The three items were 1) "The patient sees objects or animals even though there is nothing there", 2) "The patient is troubled by hearing voices in her/his head", and 3) "The patient declares that she/he hears a voice speaking aloud". The three items were assessed, during a phone interview, by the caregiver with the help of a five-point Likert scale: zero $=$ certainly does not apply to the patient, one $=$ possibly does not apply to the patient, two $=$ unsure, three $=$ possibly applies to the patient, four $=$ certainly applies to the patient. The assessment during the lockdown occurred from March 26th 2020 to November 27th 2020. Note that during this period the lockdown was implemented in France with some eased restrictions from July to September 2020, although these eased restrictions mainly concerned the general population and not residents of retirement homes.

\section{Results}

To test our hypothesis, we compared scores of hallucinations before and during lockdown using Wilcoxon signed rank tests. We used non-parametric tests since Shapiro-Wilk tests showed non-normal distributions of data [for data related to hallucinations before lockdown: $W(47)=0.85, p<0.001$, for data related to hallucinations during lockdown: $W(47)=0.71, p<0.001]$. We provided effect sizes $[d=0.2$ can be considered a small effect size, $d=0.5$ represents a medium effect size and $d=0.8$ refers to a large effect size [32]]. The effect size was calculated for non-parametric tests following recommendations by Rosenthal and DiMatteo [33], and Ellis [34]. For all tests, level of significance was set as $p \leq 0.05$.

\section{Increased Hallucinations During Lockdown}

As illustrated in Fig. 1, analyses demonstrated a significantly higher hallucinations score during the lockdown (Mean=3.49, Median $=4.00, S D=0.58)$ compared to before the lockdown $($ Mean $=2.96$, Median $=3.00, S D=0.81), Z=3.76, p<0.001$, Cohen's $d=1.31$. 
4

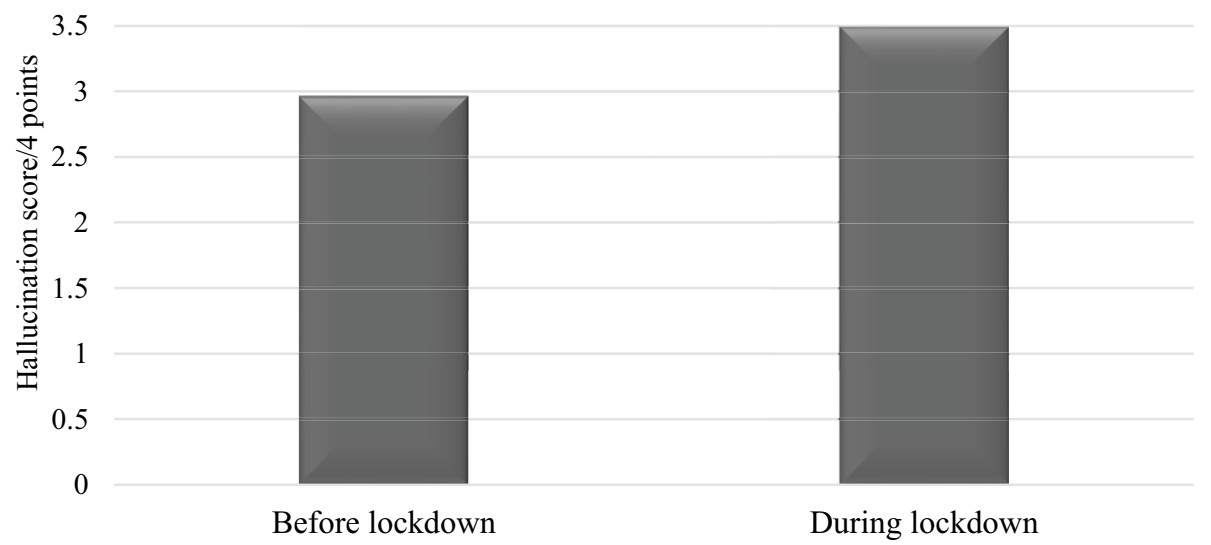

Fig. 1 Mean scores of hallucinations in AD patients before and during the lockdown

\section{Complementary Analysis}

For convenience, we assessed potential relationships between hallucination scores and scores on the Mini Mental Stat Examination using Pearson's correlations. We found no significant correlations neither between hallucination scores before the lockdown and scores on the Mini Mental Stat Examination $(r=-0.18, p=0.23)$, nor between hallucination scores during the lockdown and scores on the Mini Mental Stat Examination $(r=-0.14, p=0.36)$. Significant correlations were observed between hallucinations before and during the lockdown $(r=0.45, p=0.001)$.

\section{Discussion}

We assessed hallucinations in patients with $\mathrm{AD}$ who lived in retirement homes during the lockdown. More specifically, we targeted patients with AD presenting hallucinations before the lockdown and compared their scores of hallucinations during the lockdown with their scores before the lockdown. The results demonstrated significantly higher hallucination scores during the lockdown when compared with before the lockdown.

The increased hallucinations in patients with $\mathrm{AD}$ who lived in retirement homes during the lockdown, as observed in our study, can be attributed to the restrictive measures as implemented to cope with the spread of Covid-19. In retirement homes, non-essential internal activities and even external activities involving access to health services resources (e.g., access to external memory clinics) were canceled. Basic internal social activities such as communal dining were also restricted and residents were invited not to leave their wards, and in some cases, their rooms, in order to avoid contracting the virus. In addition to these restrictions, there was also the reduced physical contact with family members. While family members were able to visit the residents after the first weeks of the lockdown, visits occurred under strict conditions. For instance, visits were generally short and only one (or very few, in case of an emergency) member of the family was allowed. During these visits, 
contact was distant (e.g., at least 1.5-m distance) and, in many cases, occurred behind plexiglass or even behind windows. During the visits, family members had to wear a protective mouth mask, decreasing the ability to communicate, even with facial expressions, with residents. Although some patients were able to communicate with family members using technology (e.g., video calls), this communication is hindered by cognitive and sensory impairments in patients with $\mathrm{AD}$, especially in those in moderate to advanced stages of the disease as was the case of many of our participants (see mean scores on the MMSE). Also, this technology can be considered as a modest substitute for physical contact as needed by patients with AD [35]. Taken together, the social distancing measures implemented during the lockdown in France to contain the spread of Covid-19 might have increased loneliness and sensory deprivation, increasing hallucinations in patients with AD living in retirement homes, especially in those suffering hallucinations prior to the lockdown.

As we tend to suggest, the restrictive measures, as implemented by retirement homes during the lockdown, might have increased social isolation, sensory deprivation, and feelings of loneliness, and consequently, hallucinatory experiences in patients with AD. More specifically, we suggest that the reduced social contacts, cognitive stimulation, and physical activity during the lockdown are conditions that contribute to sensory deprivation and feelings of loneliness in patients with $\mathrm{AD}$, increasing their hallucinations. Our assumption can be supported by research demonstrating how social distancing during lockdown has increased levels of loneliness in patients with AD [22-24]. Critically, our assumption can be supported by research demonstrating a relationship between loneliness and hallucinations in patients with $\mathrm{AD}$ [36]. According to this research, emptiness and boredom, as experienced by patients with AD suffering loneliness, may be compensated for by creating imagined stimuli, i.e., by generating hallucinatory experiences [36]. Thus, it is likely that the reduced number of social contacts, and reduced levels of cognitive stimulation and physical activity during the lockdown might have led to emptiness, boredom and increased sensory deprivation in patients with AD living in retirement homes, leading to creating imagined stimuli and generating hallucinatory experiences in these patients.

The increased occurrence of hallucinations, as observed in our study, is likely to result from the social distancing measures rather than from the cognitive decline in patients. As demonstrated by our correlational analysis, no significant differences were observed between scores of patients in the Mini Mental Stat Examination and hallucinations. Thus, cognitive decline does not seem to be involved in the hallucinatory experiences in our study. Regardless of the cognitive account, it is important to situate our study within the current knowledge on the effects of lockdown on hallucinations in AD. Our findings extend those by Cagnin et al. [28] who investigated hallucinations in patients with AD living in their own homes, and who observed the increased occurrence of hallucinations in some of the patients. This is not surprising, as patients in the Cagnin et al. [28] study were living in their own homes with their family members. Compared with the Cagnin et al. [28] study, patients in the present study had little physical contact with family members, and thus, little affective and social interactions, which may explain the increased occurrence of hallucinatory experiences in our participants. It is also worth noting that our study involved patients with AD who presented hallucinations prior the lockdown. In other words, our results demonstrate increased hallucinations during the lockdown in patients living in retirement homes and presenting hallucinations prior the lockdown. It would be of interest to compare our findings with a sample including patients with AD living in retirement homes without any hallucinatory symptomatology prior to the lockdown in order to examine whether the lockdown may also elicit an occurrence of hallucinatory in these patients as well. 
To summarize, because patients with AD have a significantly higher risk of Covid-19 infection, retirement homes were forced to implement strict restrictive measures. However, these restrictive measures have negatively impacted mental health in patients with AD. More specifically, and as demonstrated in our study, these restrictive measures have increased the occurrence of hallucinations in patients with $\mathrm{AD}$ living in retirement homes, at least in those who were already experiencing hallucinations prior to the lockdown. The decrease in social and physical activities during the lockdown, and especially the physical separation of residents from family members, may have led to decreased levels of sensory stimulation and increased levels of loneliness, leading to an increase in the occurrence of hallucinatory experiences in patients with AD living in retirement homes during the lockdown.

Acknowledgements The first author was supported by the LABEX (excellence laboratory, program investment for the future) DISTALZ (Development of Innovative Strategies for a Transdisciplinary Approach to Alzheimer Disease)

Data Availability Data will be available upon reasonable request by email to the first author.

\section{Declarations}

Research Involving Human Participants and/or Animals This study was designed and conducted in accordance with the Declaration of Helsinki.

Informed Consent Participants provided their consent to participate.

Conflicts of Interest The authors declare no conflict of interest.

\section{References}

1. El Haj M, Allain P, Annweiler C, Boutoleau-Bretonnière C, Chapelet G, Gallouj K, et al. Burnout of Healthcare Workers in Acute Care Geriatric Facilities During the COVID-19 Crisis: An Online-Based Study. J Alzheimers Dis. 2020;78:847-52.

2. McKhann G, Knopman DS, Chertkow H, Hyman BT, Jack CR Jr, Kawas CH, et al. The diagnosis of dementia due to Alzheimer's disease: recommendations from the National Institute on Aging-Alzheimer's Association workgroups on diagnostic guidelines for Alzheimer's disease. Alzheimer's Dementia. 2011;7(3):263-9.

3. El Haj M, Roche J, Jardri R, Kapogiannis D, Gallouj K, Antoine P. Clinical and neurocognitive aspects of hallucinations in Alzheimer's disease. Neurosci Biobehav Rev. 2017;83:713-20.

4. El Haj M, Badcock JC, Jardri R, Larøi F, Roche J, Sommer IE, et al. A look into hallucinations: the relationship between visual imagery and hallucinations in Alzheimer's disease. Cogn Neuropsychiatry. 2019;24(4):275-83.

5. Bassiony MM, Lyketsos CG. Delusions and hallucinations in Alzheimer's disease: review of the brain decade. Psychosomatics. 2003;44(5):388-401.

6. Linszen MMJ, Lemstra AW, Dauwan M, Brouwer RM, Scheltens P, Sommer IEC. Understanding hallucinations in probable Alzheimer's disease: Very low prevalence rates in a tertiary memory clinic. Alzheimer's \& Dementia: Diagnosis, Assessment \& Disease Monitoring. 2018.

7. El Haj M, Larøi F. Olfactory hallucinations in Alzheimer's disease. Acta Neuropsychiatrica. 2020;1-6.

8. El Haj M, Badcock JC, Larøi F. Hallucinations and source monitoring in Alzheimer's disease. Cogn Neuropsychiatry. 2020;25(6):435-46.

9. El Haj M, Gallouj K, Dehon H, Roche J, Laroi F. Hallucinations in Alzheimer's disease: failure to suppress irrelevant memories. Cogn Neuropsychiatry. 2018;23(3):142-53.

10. El Haj M, Laroi F, Gely-Nargeot MC, Raffard S. Inhibitory deterioration may contribute to hallucinations in Alzheimer's disease. Cogn Neuropsychiatry. 2015;20(4):281-95.

11. Donovan NJ, Wadsworth LP, Lorius N, Locascio JJ, Rentz DM, Johnson KA, et al. Regional cortical thinning predicts worsening apathy and hallucinations across the Alzheimer disease spectrum. Am J Geriatr Psychiatry. 2014;22(11):1168-79. 
12. Lin SH, Yu CY, Pai MC. The occipital white matter lesions in Alzheimer's disease patients with visual hallucinations. Clin Imaging. 2006;30(6):388-93.

13. Mega MS, Lee L, Dinov ID, Mishkin F, Toga AW, Cummings JL. Cerebral correlates of psychotic symptoms in Alzheimer's disease. J Neurol Neurosurg Psychiatry. 2000;69(2):167-71.

14. Aarsland D, Cummings JL, Yenner G, Miller B. Relationship of aggressive behavior to other neuropsychiatric symptoms in patients with Alzheimer's disease. Am J Psychiatry. 1996;153(2):243-7.

15. Lerner AJ, Koss E, Patterson MB, Ownby RL, Hedera P, Friedland RP, et al. Concomitants of visual hallucinations in Alzheimer's disease. Neurology. 1994;44(3 Pt 1):523-7.

16. Haupt M, Kurz A, Janner M. A 2-year follow-up of behavioural and psychological symptoms in Alzheimer's disease. Dement Geriatr Cogn Disord. 2000;11(3):147-52.

17. Scarmeas N, Brandt J, Albert M, Hadjigeorgiou G, Papadimitriou A, Dubois B, et al. Delusions and hallucinations are associated with worse outcome in Alzheimer disease. Arch Neurol. 2005;62(10):1601-8.

18. Weamer EA, Emanuel JE, Varon D, Miyahara S, Wilkosz PA, Lopez OL, et al. The relationship of excess cognitive impairment in MCI and early Alzheimer's disease to the subsequent emergence of psychosis. Int Psychogeriatr. 2009;21(1):78-85.

19. Ornstein K, Gaugler JE, Devanand DP, Scarmeas N, Zhu C, Stern Y. The differential impact of unique behavioral and psychological symptoms for the dementia caregiver: how and why do patients' individual symptom clusters impact caregiver depressive symptoms? Am J Geriatr Psychiatry. 2013;21(12):1277-86.

20. Rocca P, Leotta D, Liffredo C, Mingrone C, Sigaudo M, Capellero B, et al. Neuropsychiatric symptoms underlying caregiver stress and insight in Alzheimer's disease. Dement Geriatr Cogn Disord. 2010;30(1):57-63.

21. Boutoleau-Bretonnière C, Pouclet-Courtemanche H, Gillet A, Bernard A, Deruet AL, Gouraud I, et al. The Effects of Confinement on Neuropsychiatric Symptoms in Alzheimer's Disease During the COVID-19 Crisis. J Alzheimers Dis. 2020;76:41-7.

22. Giebel C, Cannon J, Hanna K, Butchard S, Eley R, Gaughan A, et al. Impact of COVID-19 related social support service closures on people with dementia and unpaid carers: a qualitative study. Aging Ment Health. 2020;1-8.

23. Verbeek H, Gerritsen DL, Backhaus R, de Boer BS, Koopmans R, Hamers JPH. Allowing Visitors Back in the Nursing Home During the COVID-19 Crisis: A Dutch National Study Into First Experiences and Impact on Well-Being. J Am Med Dir Assoc. 2020;21(7):900-4.

24. Van der Roest HG, Prins M, van der Velden C, Steinmetz S, Stolte E, van Tilburg TG, et al. The Impact of COVID-19 Measures on Well-Being of Older Long-Term Care Facility Residents in the Netherlands. J Am Med Dir Assoc. 2020;21(11):1569-70.

25. El Haj M, Altintas E, Chapelet G, Kapogiannis D, Gallouj K. High depression and anxiety in people with Alzheimer's disease living in retirement homes during the Covid-19 crisis. Psychiatry Res. 2020;113294.

26. El Haj M, Larøi F, Gallouj K. Hallucinations in a Patient with Alzheimer's Disease During the COVID-19 Crisis: A Case Study. J Alzheimers Dis Rep. 2020;4:455-8.

27. EL Haj M, Laroi F. Increased hallucinations in patients with Alzheimer's disease during the Covid19-lockdown: A presentation of two cases. In: Moustafa AA, editor. Pandemic Mental Health: Coping in a COVID-19 Environment. The Netherlands: Elsevier; Accepted. 2022.

28. Cagnin A, Di Lorenzo R, Marra C, Bonanni L, Cupidi C, Lagana V, et al. Behavioral and Psychological Effects of Coronavirus Disease-19 Quarantine in Patients With Dementia. Front Psych. 2020;11:578015.

29. Folstein MF, Folstein SE, McHugh PR. "Mini-mental state". A practical method for grading the cognitive state of patients for the clinician. J Psychiatr Res. 1975;12(3):189-98.

30. Faul F, Erdfelder E, Lang AG, Buchner A. G*Power 3: a flexible statistical power analysis program for the social, behavioral, and biomedical sciences. Behav Res Methods. 2007;39(2):175-91.

31. Cohen J. Statistical power analysis current directions. Psychol Sci. 1992;1(3):98-101.

32. Cohen J. Statistical power analysis for the behavioral sciences. Hillsdale, NJ: Erlbaum Associates. 1988.

33. Rosenthal R, DiMatteo MR. Meta-analysis: recent developments in quantitative methods for literature reviews. Annu Rev Psychol. 2001;52:59-82.

34. Ellis PD. The Essential Guide to Effect Sizes: Statistical Power, Meta-Analysis, and the Interpretation of Research Results. New York, NY: Cambridge University Press; 2010.

35. Gleeson M, Timmins F. Touch: a fundamental aspect of communication with older people experiencing dementia. Nurs Older People. 2004;16(2):18-21.

36. El Haj M, Jardri R, Laroi F, Antoine P. Hallucinations, loneliness, and social isolation in Alzheimer's disease. Cogn Neuropsychiatry. 2016;21(1):1-13.

Publisher's Note Springer Nature remains neutral with regard to jurisdictional claims in published maps and institutional affiliations. 
Mohamad El Haj is neuropsychologist full professor of cognitive psychology at the University of Nantes France. He dedicates his clinical and research activity to cognitive dysfunction in Alzheimer's Disease.

Frank Larøi is Professor of clinical psychology at the University of Bergen Sweden. He dedicates his research activity to the study of hallucinations.

Karim Gallouj is geriatrician at the hospital of Tourcoing France. He dedicates his clinical activity to geriatric dysfunction. 